\title{
In Verteidigung der Rückhaltlosigkeit der menschlichen Geburt
}

\author{
Kommentar zu „,Die Auswahl zukünftiger Kinder“ von \\ Tatjana Tarkian
}

\section{Olivia Mitscherlich-Schönherr}

Online publiziert: 15 . Juni 2020

(C) Der/die Autor(en) 2020

Zusammenfassung In meinem Kommentar gehe ich von dem Verständnis der menschlichen Geburt in der Moderne aus, das Tatjana Tarkian mit vielen teilt: dem Verständnis, dass die menschliche Geburt in der Moderne ein autonom plan- und durchführbares „Projekt“ (Tarkian, Abschnitt 5) bilde. Im ersten Schritt meiner Überlegungen zeige ich, dass diese Vorstellung reduktionistisch ist: sie blendet sowohl die Widerfahrensaspekte als auch die dialogische Anlage der Geburt ab. In den weiteren Schritten meiner Überlegungen skizziere ich, dass Tarkians reduktionistisches Verständnis der menschlichen Geburt auf die Modulation ihrer Forschungsfrage und auf ihr normatives Leitprinzip durchschlägt - und dort eigene Abstraktionen hervorruft. Nur da Tarkian die Geburt als „Projekt“ der eigenen Reproduktion versteht, können sich ihr die Entscheidungen, zu denen pränatalmedizinische Tests befähigen, als „Auswahl“ zwischen „,gesunden Kindern“ und „Kindern mit gesundheitlichen Beeinträchtigungen oder Normabweichungen“ (Tarkian, Abschnitt 3) darstellen. Und nur da sie die Geburt in Analogie zu anderen „Lebensprojekten“ versteht, kann sie ihr Leitprinzip der Freiheit im Gebären als reproduktive Handlungsfreiheit deuten um zu diskutieren, ob die reproduktive Handlungsfreiheit beim Gebrauch pränatalmedizinischer Angebote an Grenzen stoße, da sie Anderen schade.

Tatjana Tarkians Überlegungen kommt ein großer Verdienst zu: sie setzen zu einer ernsthaften, philosophisch geführten Auseinandersetzung mit den Fragen der genetischen Testung und pränatalen Selektion instand. Wenn ich im Folgenden grundlegende Einwände gegen Tarkians Position erhebe, so zeugt dies nicht zuletzt von meinem Respekt vor ihren Bemühungen um eine genuin philosophische Positionierung in den ansonsten oft stark ideologisch geführten Debatten über die diagnostischen

O. Mitscherlich-Schönherr $(\bowtie)$

Hochschule für Philosophie München, Kaulbachstraße 31a, 80539 München, Deutschland

E-Mail: olivia.mitscherlich@hfph.de 
und therapeutischen Möglichkeiten der Pränatalmedizin. ${ }^{1}$ In meinen Reaktionen auf Tarkian geht es mir um eine an der Sache der modernen Geburt unter den Bedingungen der Pränatalmedizin orientierten Auseinandersetzung aus den Perspektiven unterschiedlicher, philosophischer Theorietraditionen. Tarkian wird - hoffentlich ebenso viele Rückfragen an meine Überlegungen haben wie ich an die ihrigen.

Tarkian stellt ihre Überlegungen unter die Frage, ob die - durch genetische Testung eröffnete - „Auswahl zukünftiger Kinder“ ethisch zulässig sei. Solch eine „Auswahl“" versteht sie als die „Auswahl von gesunden Kindern anstelle von Kindern mit gesundheitlichen Beeinträchtigungen oder Normabweichungen, wie sie eine geistige Behinderung darstellt“ (Tarkian, Abschnitt 3). In der ethischen Auseinandersetzung mit solcher Wahl nimmt sie am „Prinzip der reproduktiven Freiheit“ (Tarkian, Abschnitt 4) Maß, um „Freiheitsspielräume und Privatsphäre in Fragen der Fortpflanzung [zu verteidigen], wenn nicht gewichtige moralische Gründe aufgezeigt werden können, welche den Handlungen von Personen Grenzen setzen“" (Ebd.). In der Formulierung ihrer Frage und ihres ethischen Leitprinzips nimmt Tarkian einen spezifischen „Reflexionsbegriff“ der modernen Geburt in Anspruch: dass die menschliche Geburt in der Moderne ein autonom plan- und durchführbares „Projekt“ (Tarkian, Abschnitt 5) sei. Da diese Vorstellung über eine moderne Geburt in der Gegenwart sehr verbreitet ist, setze ich im ersten Abschnitt meines Kommentars hier an, um ihre Reduktionismen aufzuzeigen. In den Abschnitten zwei und drei werde ich mich Tarkians Fragestellung und normativem Leitprinzip zuwenden, um deren Abhängigkeit von dieser abstrakten Anthropologie der Geburt und die daraus resultierenden Probleme zu skizzieren. Im abschließenden vierten Abschnitt werde ich die Schwierigkeiten von Tarkians normativem Leitprinzip an ihrer Position zu Abbrüchen einer Schwangerschaft mit einem Kind diskutieren, an dem durch genetische Testung eine schwere Erkrankung oder Behinderung diagnostiziert wurde.

\section{Der Reduktionismus der Auffassung von der Geburt als „Kette von Ereignissen“6}

Ihre Vorstellung von der modernen Geburt klingt bei Tarkian nur nebenbei - im Rahmen ihrer geschichtsphilosophischen Ausgangshypothese - an. In Anschluss an Allan Buchanan und Dieter Birnbacher vertritt sie die geschichtsphilosophische These, dass die Geburt in der Moderne eine Entwicklung ,von der Widerfahrnis zur Gestaltung, von der Kontingenz zum Arrangement, von der Heteronomie zur Autonomie“ (Tarkian, Abschnitt 1) durchlaufen habe, bzw. dass sie sich ,mit dem Fortschritt in der Reproduktionsmedizin, Humangenetik und vorgeburtlichen Diagnostik [...] zunehmend vom zufälligen Ereignis zum Ergebnis einer Kette von Entscheidungen gewandelt“ (Ebd.) habe. Das gegenwärtige Gebären stellt sich ihr

\footnotetext{
1 In meinen Überlegungen zehre ich von Diskussionen über eine gelingende Geburt, die ich mit vielen KollegInnen habe führen dürfen. Ein Abbild dieser Diskussionen liefert der Sammelband Die gelingende Geburt. Zeitgenössische Theorien im interdisziplinären Dialog, der im Herbst 2020 unter der Herausgeberschaft von Reiner Anselm und mir erscheinen wird. An den entsprechenden Stellen verweise ich auf die Beiträge in diesem Band, von denen ich gelernt habe.
} 
darin als „Projekt“ (Tarkian, Abschnitt 5) der Reproduktion bzw. der Familiengründung dar, das autonom geplant und durchgeführt werden könne. Meines Erachtens vertritt sie darin eine subjektivistische Anthropologie, die die menschliche Geburt sowohl in inhaltlicher als auch in formaler Hinsicht verkürzt.

Die inhaltlichen Verkürzungen, denen der Gedanke von der Geburt als einem „Projekt“ aufsitzt, werden mit Blick auf ein empirisches Faktum des gegenwärtigen Gebärens offenbar: dass die Chancen für eine erfolgreiche Schwangerschaft bei künstlicher Befruchtung in vitro weit schlechter stehen als bei einer natürlichen Befruchtung in uteru. Auch unter den Bedingungen der fortschrittlichsten Methoden zu ihrer technischen Gestaltung bleibt die menschliche Geburt folglich immer auch ein Widerfahrnis. Indem Tarkian, Buchanan und Birnbacher von der Tilgung des Widerfahrnischarakters im modernen Gebären ausgehen, sitzen sie dem normativen „Leitbild“ (Kersting 2017) der modernen Reproduktionsmedizin auf: dass die Geburt ein „Projekt“ der Reproduktion sein soll, das sich planen und in den verschiedenen Schritten seiner Verwirklichung autonom steuern und gestalten lässt. Mit dieser Auffassung gleicht Tarkian die moderne Geburt Projekten an, die wir inmitten des Lebens überschauen und realisieren können. Zugleich blendet sie aus, dass all unsere Gestaltungsentscheidungen über die Ausgestaltung unseres Gebärens in umfassende Prozesse des Gebärens integriert sind, die nicht nur die Art unseres Gebärens grundlegend mitbestimmen, sondern vielmehr auch darüber bestimmen, ob wir überhaupt Kinder kriegen.

Dem subjektivistisch verkürzten Verständnis der Geburt als einem „Projekt“ der Reproduktion, das in einer „Kette von Entscheidungen“ umgesetzt wird, möchte ich einen ganzheitlichen Begriff der genuin personalen Geburt entgegensetzen. Dabei gehe ich wie Tarkian davon aus, dass sich die Geburt nicht auf das Ereignis der Entbindung beschränken lässt, sondern vielmehr einen Prozess darstellt, der mit der Zeugung beginnt (und über die Entbindung hinausreicht). Unter unserer personalen Lebensform $^{2}$ greifen in der Geburt die unterschiedlichen Aspekte unseres menschlichen Lebens ineinander: neben unseren individuellen und sozio-kulturell geteilten Gestaltungspraktiken sind das biologische Prozesse, unmittelbare, leibliche und seelische Erfahrungen sowie sozio-kulturell tradierte Bilder. ${ }^{3}$ Auch in der modernen Ausgestaltung der personalen Geburt verschränken sich damit passives Widerfahrnis und aktive Gestaltung. Hinzu kommen allein neue technische Gestaltungsmöglichkeiten: Möglichkeiten der pränatalmedizinischen Testung und Manipulation, die den Widerfahrnischarakter der Geburt jedoch nicht tilgen.

Vor dem Hintergrund dieses personalen Verständnisses der Geburt tritt die weitere Verkürzung hervor, die Tarkians Vorstellung über die moderne Geburt in formaler Hinsicht bestimmt: mit ihrer Auffassung von der Geburt als einem Projekt, das in einer Kette von Entscheidungen umgesetzt werde, reduziert sie die dialogische Form der modernen Geburt auf ein monologisches Unternehmen. Tarkian

\footnotetext{
2 Beim Verständnis unserer personalen Lebensform greife ich auf einen Gedanken von Helmuth Plessner zurück; vgl. Plessner 1975, 288-346.

3 Ausführlicher entwickle ich dieses Verständnis des genuin personalen Gebärens in dem Aufsatz ,Das Lieben in der Geburt. Eine verstehende Liebesethik des Zur-Welt-Bringens von Kindern“, den ich meinerseits zu dem von Reiner Anselm und mir organisierten Band Die gelingende Geburt (i. V.) beitrage.
} 
unterscheidet die tatsächliche Beziehung zwischen ,aktualen Eltern“ und ihren ,geborenen Kindern“ (Tarkian, Abschnitt 5; Herv. OMS) von dem Verhältnis zwischen „potenziellen Eltern“ (ebd.) und ihren ,zukünftige[n] (mögliche[n]) Kind[ern]“ (Tarkian, Abschnitt 3) am Beginn des „Projekt[s]“ der „Familiengründung“ (ebd.). In Bezug auf die letztere betont sie: „Mögliche Kinder sind kein Gegenüber“ (Tarkian, Abschnitt 5). Implizit geht sie darin davon aus, dass erst die Entbindung - als das Ereignis, in dem das Kind für alle sichtbar in der Welt in Erscheinung tritt das Eltern-Kind-Verhältnis in ein Beziehungsverhältnis transformiere. Unter unserer personalen Lebensform ist das Gebären allerdings bereits vor dem Ereignis der Entbindung ein „Beziehungsgeschehen“ (vgl. Schües 2003; in Vorbereitung).

$\mathrm{Ab}$ einen bestimmten Zeitpunkt während der Schwangerschaft tritt das Kind der werdenden Mutter als „Gegenüber“ entgegen. Indem es Gefühlszustände in Gestalt seiner Bewegungen zum Ausdruck bringt und die Schwangere durch Veränderungen ihres Körperleibes auf das kindliche Ausdrucksverhalten antworten kann, können Mutter und Kind erste, leibliche Formen einer dialogischen Beziehung ausüben. Aber bereits vor diesem Zeitpunkt der Begegnung bestehen in beiden Stadien der Geburt, mit denen sich Tarkian auseinandersetzt, basale Formen der Beziehung: sowohl zu Beginn der Schwangerschaft als auch in dem Zeugen, das gegenwärtig in Deutschland mit Hilfe von Praktiken der künstlichen Befruchtung bzw. der In VitroFertilisation (IVF) ausgeübt wird.

Die Schwangerschaft ist von einer besonderen Form der körper-leiblichen Einheit zwischen Mutter und Kind bestimmt (vgl. Staehler 2016; in Vorbereitung). Schwangere sind keine bloß ,ppotenziellen Eltern“ von „möglichen“ Kindern - wie Tarkian suggeriert; vielmehr sind sie ,aktual“" werdende Mütter ihres zukünftigen Kindes, bzw. im Fall von Mehrlingsschwangerschaften ihrer zukünftigen Kinder. Die Aktualität des Mutter-Werdens drückt sich nicht nur in den grundlegenden Veränderungen ihres Körper-Leibes aus, die Schwangere durchlaufen (vgl. Staehler in Vorbereitung), an sie knüpfen sich auch eine Reihe von Rechten; und ihr künftiges MutterSein kann von der Schwangeren - im Unterschied zu ihrem künftigen Schulspielerin-Sein - willentlich nicht durch das einfache Verwerfen dieser Zukunft, sondern allein durch einen medizinischen Eingriff verhindert werden. Komplementär dazu ist der Embryo bereits am Beginn der Schwangerschaft kein „mögliches“ Kind, dem erst durch die Entbindung als einem Herstellungsakt Wirklichkeit verschafft würde; er ist vielmehr ein wirkliches, im Entstehen begriffenes Kind, das im körperleiblichen Miteinander mit der Mutter eine Entwicklung durchläuft, die seinem Aufdie-Welt-Kommen zustrebt, das mittels pränataldiagnostischer Verfahren untersucht werden kann und unter besonderen Schutzrechten steht.

Bereits in den Formen des Zeugens, die gegenwärtig in Deutschland im Zuge der Praktiken der künstlichen Befruchtung ausgeübt werden (dürfen), wird eine basale Form der Beziehung zwischen der Frau und dem künstlich erzeugten Embryo etabliert (vgl. Wiesemann 2006, 57 ff.). Diese Beziehung wird nicht nur subjektiv als ein Sich-Beziehen der Mutter auf den künstlich erzeugten Embryo im Modus von Ängsten, Hoffnungen, Phantasmen und Sehnsüchten hergestellt. Sie hat zugleich ein biologisches Pendant, indem der Embryo aus der Eizelle der Frau erzeugt wird. Und sie findet in Deutschland in einem - vom Embryonenschutzgesetz gestifteten Rechtsrahmen statt, der die biologische und soziale Dimension der künstlichen Be- 
fruchtung zusammenhält: einem Rechtsrahmen, unter dem nicht nur Embryonen in vitro erzeugt werden, sondern unter dem zugleich auch die Frau, der die künstlich befruchteten Eizellen entnommen und der der künstlich erzeugte Embryo eingesetzt wird, dazu befähigt wird, sich auf ebendiesen Embryo als ,ihren“ Embryo zu beziehen. Eine Entkoppelung der technisch-biologischen Befruchtungsprozesse von den sozialen Dimensionen der Zeugung verhindert das Embryonenschutzgesetz: es verbietet Eizellspende und Leihmutterschaft und es legt in Bezug auf die IVF-Verfahren fest, dass nicht mehr als drei Eizellen pro Zyklus befruchtet und dabei nicht mehr Embryonen hergestellt werden dürfen, als einer Frau auch tatsächlich übertragen werden sollen. Genauso wie Schwangere zu Beginn ihrer Schwangerschaft sind folglich auch Frauen, die sich gegenwärtig in Deutschland einer IVF-Behandlung unterziehen, keine bloß ,potenziellen“ Mütter von „möglichen“ Kindern, sondern bereits ,aktual“" werdende Mütter der Embryonen, die aus ihren Eizellen erzeugt und ihnen transferiert werden - so ungewiss und gefährdet der weitere Verlauf ihrer Mutter-Kind-Beziehung in beiden Fällen auch noch sein mag. Von der Aktualität ihrer Existenz als werdender Mutter zeugt nicht zuletzt das Leiden, das Frauen häufig erleben, wenn innerhalb ihrer Beziehung zu ihrem in vitro erzeugten Embryo der Schritt in ein leiblich geteiltes Miteinander durch dessen Transfer in den eigenen Körperleib nicht gelingt. Komplementär dazu ist wiederum der in vitro erzeugte Embryo, der einer Frau transferiert werden soll, nicht nur ein „mögliches“, sondern vielmehr ein wirkliches, im Entstehen begriffenes Kind, das aus den Ei- und Samenzellen bestimmter Menschen erzeugt wurde, mittels präimplantationsdiagnostischer Verfahren untersucht werden kann und unter besonderen Schutzrechten steht. Allein auf ein besonderes Dasein, das innerhalb der gegenwärtig in Deutschland praktizierten IVF hervorgebracht wird, passt meiner Ansicht nach die Statusbestimmung als ein mögliches Kind: auf die ,verwaisten Embryonen“. Die ,verwaisten Embryonen“ fallen aus den sozialen Beziehungsdimensionen der Zeugung heraus, indem sie in vitro erzeugt worden sind, jedoch nicht mehr transferiert werden sollen, da ein Transfer mit einem anderen Embryo bereits erfolgreich war. Auch ihnen kommt jedoch nicht der Status als ,zukünftige[n] (mögliche[n]) Kinder[n]“ zu, den Tarkian allen Kindern vor der Geburt zuspricht. „Verwaiste Embryonen“ sind weder aktual noch potenzial zukünftige Kinder, sondern potenzielle Kinder ohne Zukunft.

\section{Die Abstraktheit der Vorstellung einer von der gegenwärtigen Reproduktionsmedizin eröffneten Wahl zwischen möglichen, zukünftigen Kindern}

Ihr abstraktes Verständnis von der Geburt als einem monologischen „Projekt“, das sich in einer „Kette von Entscheidungen“ autonom planen und realisieren lasse, stellt die Weichen für Tarkians Auseinandersetzung mit den Entscheidungen, zu denen uns die modernen Möglichkeiten der pränatalen Diagnostik und Therapie befähigen. Es bestimmt bereits ihre Fragestellung bzw. die Modulation ihres Forschungsgegenstandes. Nur weil sie die Geburt in Analogie zu anderen „Projekten“ versteht, die wir im Leben verfolgen, kann Tarkian die Entscheidungen, zu denen pränatalmedizinische Tests befähigen, als „Auswahl“ zwischen ,gesunden Kindern“ 
und „Kindern mit gesundheitlichen Beeinträchtigungen oder Normabweichungen“ (a.a.O) bzw. als „Versuch“ begreifen, ,ein (mögliches) zukünftiges Kind durch ein anderes (mögliches) zukünftiges Kind zu ,ersetzen““(S. 4) - um dann nach der ethischen Zulässigkeit solcher Unternehmungen zu fragen.

Diese Problemformulierung geht meines Erachtens in zwei Hinsichten am Phänomen der Entscheidungen vorbei, die in der Gegenwart in Deutschland tatsächlich gefällt werden. Vor dem Hintergrund meiner obigen Überlegungen möchte ich zum einen betonen, dass das Kind, mit dem das Leben fortgeführt werden soll oder nicht, im Beziehungsgefüge einer Schwangerschaft bzw. einer künstlichen Befruchtung für die Frau kein ,(mögliches) zukünftiges Kind“ ist. Für die werdende Mutter ist ihr Embryo - in uteru oder in vitro - nicht deshalb zukünftig, da letzteres in der Gegenwart allein als eine Möglichkeit da ist, die sie selbst durch Herstellungsakte in den anderen Seins-Status der Wirklichkeit überführen müsste. Der Embryo ist für sie vielmehr insofern ihr zukünftiges Kind, als sie sich auf ihn in der Gegenwart im Lichte ihrer künftigen Beziehung bezieht: im Lichte ihres künftigen, ganzheitlich geteilten Lebens, in dem ihr das Kind auf vielfältige Weise in seiner körperleiblichen Erscheinung, seinem leiblichen und sprachlichen Ausdrucksverhalten als Gegenüber entgegentreten wird.

Zum anderen ist Tarkians Problemformulierung meines Erachtens schief, da die Entscheidungen, zu denen Eltern durch die gegenwärtig in Deutschland erlaubten und praktizierten Tests befähigt werden, keine Akte der „Auswahl“ bzw. der Ersetzung zwischen zur Wahl stehenden, möglichen Kindern, sondern vielmehr genuine Beziehungsentscheidungen darstellen: Entscheidungen über die künftige Fortsetzung der basalen Beziehung, die die Mutter (bzw. die Eltern) zum Kind aktual im Zuge der Schwangerschaft bzw. der Zeugung mittels IVF unterhält (bzw. unterhalten). Dies sei knapp in Bezug auf beide Formen der pränatalmedizinischen Testung skizziert, die gegenwärtig in Deutschland erlaubt sind und ausgeübt werden: die „Präimplantationsdiagnostik“ (PID) und die „Pränataldiagnostik“ (PND).

Das Embryonenschutzgesetz stiftet in Deutschland einen Rechtsrahmen, der die biologische und soziale Dimension der künstlichen Befruchtung auch unter der Anwendung von PID am in vitro erzeugten Embryo zusammenhält. Die Gefahr einer Entkoppelung der technisch-biologischen Befruchtungsprozesse von der Beziehungsdimension durch die Anwendung von PID wendet es ab, indem es strenge Auflagen an die Testung der in vitro erzeugten Embryonen knüpft. PID an in vitro erzeugten Embryonen ist gegenwärtig in Deutschland nur unter der Bedingung zulässig, dass ein oder beide Elternteile Anlageträger_in einer schwerwiegenden Erberkrankung sind oder ein hohes Risiko für Fehl- oder Totgeburten haben. PID eröffnet der Mutter bzw. den Eltern die Möglichkeit, sich gegen den Transfer eines bestimmten Embryos zu entscheiden, das der Frau eigentlich hätte übertragen werden sollen, da dieser bestimmte Embryo schwerwiegend erkrankt ist oder ein hohes Fehl- oder Totgeburtsrisiko trägt. Damit sind bereits die Entscheidungen, zu denen Mütter bzw. Eltern gegenwärtig in Deutschland durch PID befähigt werden, keine Wahl-, sondern Beziehungsentscheidungen.

Bei den Entscheidungen, zu denen die - in Deutschland aktuell zugelassenen Tests der PND an Embryonen in uteru befähigen, tritt dieser Status als Beziehungsentscheidung noch deutlicher hervor. Während die Rückbindung der biologischen 
Befruchtungsprozesse an die Beziehungsaspekte des Gebärens im Fall der künstlichen Befruchtung in vitro rechtlich durch das Embryonenschutzgesetz sichergestellt wird, ist sie im Fall der natürlichen Befruchtung in uteru durch unsere personale Lebensform etabliert. Damit finden die Tests an einem Embryo oder Fötus im Mutterleib mit PND inmitten der körper-leiblichen, seelischen, geistigen Beziehung der Schwangeren zu ihrem Kind statt. Inmitten dieser Beziehung können die Tests werdenden Müttern die Entscheidungen eröffnen, das Leben, das sie in Gestalt ihrer Schwangerschaft mit diesem Kind teilen, auf unterschiedliche Weise fortzusetzen, zu gestalten, oder auch abzubrechen. Die pränataldiagnostischen Tests befähigen die werdende Mutter dagegen auch deswegen nicht zu einer „Auswahl von gesunden Kindern anstelle von Kindern mit gesundheitlichen Beeinträchtigungen oder Normabweichungen, wie sie eine geistige Behinderung darstellt“" (Tarkian, Abschnitt 3), da zum Zeitpunkt der Testung völlig ungewiss ist, ob sie nach der gegenwärtigen Schwangerschaft noch einmal schwanger werden wird. Zum Zeitpunkt der Testung kommt damit einem anderen Kind tatsächlich nur der Status bloßer Möglichkeit zu - während die Schwangere hier und jetzt über den weiteren Verlauf der wirklichen Beziehung mit dem wirklichen Kind in ihrem Bauch zu entscheiden hat.

Wenn überhaupt, dann passt Tarkians Frage nach einer „Auswahl“ bzw. nach einem „Versuch, ein (mögliches) zukünftiges Kind durch ein anderes (mögliches) zukünftiges Kind zu ,ersetzen““, allein auf Entscheidungen, zu denen Frauen durch Anwendungen der PND im Rahmen von IVF befähigt würden, die gegenwärtig in Deutschland verboten sind: auf Anwendungen von PND im Rahmen von IVF, bei denen die biologisch-technischen Zeugungsprozesse vom Beziehungsaspekt entkoppelt werden, indem mehr Embryonen hergestellt werden als übertragen werden sollen - um beim Transfer ein möglichst breites „Angebot“ an Embryonen zur Verfügung zu haben, die mittels IVF auf alle möglichen Erkrankungen oder Behinderungen getestet werden sollen. Wenn Tarkian diese Unterschiede schleift und die elterlichen Entscheidungen, zu denen pränatalmedizinische Tests befähigen, just im Ausgang von diesen letztgenannten Entscheidungen versteht, dann ist sie darin ihrem abstrakten Verständnis modernen Gebärens verschuldet. Nur weil sie die widerfahrenden Aspekte an der Geburt ausblendet, inmitten derer wir unsere Gestaltungsentscheidungen treffen, können sich ihr die unterschiedlichen Entscheidungen, zu denen uns pränatalmedizinische Tests befähigen, durchgängig als ,Auswahl“ darstellen: als eine „Auswahl“, die wir scheinbar im luftleeren Raum unserer Reproduktionsprojekte ausüben.

\section{Der Reduktionismus des Leitprinzips reproduktiver Handlungsfreiheit}

Den Bezug zwischen ihrer Auffassung von der Geburt als einem autonom plan- und durchführbaren „Projekt“ und ihrem normativen Leitprinzip stellt Tarkian selbst her. Um die ethische Zulässigkeit der „Auswahl“ zwischen zwei „möglichen“ Kindern zu beurteilen, nimmt sie an einem ,liberale[n] Prinzip der reproduktiven Freiheit“ (Tarkian, Abschnitt 4) Maß. Bei dessen Einführung geht sie davon aus, ,dass es gut für den Menschen ist, Lebenspläne zu entwerfen, selbstbestimmt Entscheidungen 
$\mathrm{zu}$ treffen und sein eigenes Leben gestalten oder wenigstens auf seinen Verlauf Einfluss nehmen zu können“ (ebd.). Sie rechnet „die Entscheidung für Kinder“ (ebd.) zu diesen Lebensplänen und folgert hieraus, dass es ,gut [ist], Handlungsspielräume in Fragen zu haben, welche die Fortpflanzung betreffen“ (ebd.). „Strittig“ ist für Tarkian ,nur die Frage nach der Reichweite der reproduktiven Freiheit“ (ebd.). Meines Erachtens ist noch ein weiterer, grundlegenderer Punkt strittig: nämlich die Frage, ob aus dem Umstand, dass es gut für Menschen ist, Handlungsspielräume in Fragen der Fortpflanzung zu haben, folgt, dass wir unsere ethische Auseinandersetzung mit der Frage nach unserer Freiheit im Gebären am normativen Leitprinzip der reproduktiven Freiheit orientieren sollten.

Mit dem Gedanken der reproduktiven Freiheit stellt Tarkian ihre Überlegungen unter ein Prinzip der Handlungsfreiheit. Sie geht davon aus, dass autonome Planung und Umsetzung das eigene Gebären frei werden lassen. PND und PID stellen sich ihr als mögliche Formen der Erweiterung der reproduktiven Handlungsfreiheit dar. Unter der Voraussetzung, dass autonomes Planen und Gestalten unser Gebären frei machen, diskutiert sie allein die Frage, ob die reproduktive Freiheit beim Gebrauch pränatalmedizinischer Angebote an Grenzen stoße, da sie Anderen schade. Bei ihrer Deutung der Freiheit im Gebären als Handlungsfreiheit unterschätzt sie meines Erachtens jedoch die emotionalen Abhängigkeiten, die unser Wollen im Gebären fesseln können.

Als Quelle der Abhängigkeit im Gebären nimmt Tarkian - zurecht - die Beschneidung unserer Handlungsspielräume durch Andere in den Blick. Unsere reproduktive Freiheit sieht sie dann als gefährdet an, wenn wir in unserem Gebären von fremden Vorstellungen über ein gutes Leben und Gebären abhängen: von Vorstellungen, die uns daran hindern, unsere individuellen Vorstellungen guter Familiengründung zu realisieren (vgl. Tarkian, Abschnitt 6). Als ein drastisches Beispiel der Beschneidung von Handlungsfreiheit im Gebären wäre etwa an die politischen Maßnahmen der Bevölkerungspolitik zu denken, die in China bis heute vorgenommen werden (vgl. https://www.igfm.de/china-ein-kind-politik/; letzter Aufruf März 2020). In diesem Sinne will Tarkian die - durch genetische Testung ermöglichte - Auswahl künftiger Kinder denn auch „,nur dann als Erweiterung der reproduktiven Freiheit verstehen, wenn die individuelle Entscheidung zur Diagnostik selbstbestimmt und nicht unter dem Druck von verbreiteten diskriminierenden Urteilen über das Leben von Menschen mit Behinderung getroffen wird" (ebd.).

In ihrer Fokussierung auf die Handlungsfreiheit im Gebären entgehen Tarkian die Abhängigkeiten, in die unser Wollen verstrickt sein kann: die emotionalen Abhängigkeiten von uns selbst, die unser Wollen binden können, auch wenn unser Handeln keiner Fremdbestimmung durch Andere unterliegt. Im Hintergrund von Tarkians Verkürzung der Freiheit im Gebären auf Handlungsfreiheit - unter Abstraktion der Willensfreiheit - steht wiederum ihr abstrakter Begriff der Geburt. Wie oben ausgeführt, blendet Tarkian in ihrer Vorstellung vom modernen Gebären als autonom plan- und durchführbaren Projekt den Widerfahrnischarakter ab. Dabei ist nicht der Widerfahrnischarakter der Geburt als solcher, sondern vielmehr genauer die Zeitlichkeit der eigenen Lebens- und Geburtsgestaltung für die Frage nach der Freiheit im Gebären von Belang. Die Verwirklichung eigener Projekte der Fortpflanzung mag die jeweiligen Akte des Gebärens zwar zu ,den eigenen“ machen; die Freiheit des 
eigenen Wollens ist damit jedoch nicht sichergestellt. Indem ich mich an meinen eigenen Idealen der Familiengründung orientiere, kann ich vielmehr von meinem eigenen Geworden-Sein bedingt werden: von Ängsten und Ressentiments, die ich in der Vergangenheit ausgebildet habe und die mein Wollen im gegenwärtigen Gebären festlegen und auf die aktuellen Handlungen durchschlagen, in denen ich mein Gebären (mit)gestalte. Es würde zu kurz greifen, diese emotionalen Verstrickungen als Bedingtheiten von außen aufzufassen. Ich kann auch vor dem Hintergrund meiner eigenen Vergangenheit - etwa der mehrfachen Erfahrung von Schwangerschaftsabgängen - in Ängste gebannt sein, die mein gegenwärtiges Wollen in der aktuellen Schwangerschaft beherrschen und mich in Gestaltungsfragen übersehen lassen, worum es hier und jetzt für mich eigentlich ginge. ${ }^{4}$ Zusammen mit den emotionalen Verstrickungen im Gebären übersieht Tarkian das zentrale, ethische Problem, wie wir inmitten des Gebärens vor dem Hintergrund unseres individuellen Geworden-Seins in unserem Wollen frei sein können.

\section{Die Dialektiken einer Erweiterung von reproduktiver Handlungsfreiheit durch embryopathisch begründete Schwangerschaftsabbrüche}

Im letzten Schritt meiner Überlegungen möchte ich auf die Schwangerschaftsabbrüche blicken, die ausgeübt werden, nachdem in pränataldiagnostischen Tests am Erbgut des Kindes Hinweise auf eine schwere Erkrankung oder Behinderung - etwa: Trisomie 23 - aufgefunden worden sind. Die Auseinandersetzung mit diesen Fällen ist deswegen von Interesse, da sich an ihnen erkennen lässt, wie die Erweiterung von reproduktiver Handlungsfreiheit durch PND mit einer genau benennbaren Einschränkung der Willensfreiheit einhergehen kann (nicht muss): der Verstrickheit in Ressentiments gegenüber Menschen mit Behinderung. Schwangerschaftsabbrüche, die von Ressentiments gegen Menschen mit Behinderung angeleitet sind, verletzen nämlich nicht nur die Würde des betroffenen Kindes; in ihnen sind auch die Eltern in ihrem Wollen unfrei: bedingt durch Ressentiments, die in der Vergangenheit ausgebildet worden sind und sie in der Gegenwart übersehen lassen, worum es für sie selbst individuell hier und jetzt eigentlich ginge. In diesen Schlussüberlegungen werde ich zunächst Tarkians Argumentation rekonstruieren: dass Schwangerschaftsabbrüche, die in Reaktion auf die pränatalmedizinische Diagnose einer schweren Erkrankung oder Behinderung des Kindes vorgenommen werden, unsere reproduktive Handlungsfreiheit unter der Bedingung erweitern, dass sie embryopathisch begründet sind, ohne dabei diskriminierend zu sein. In meinen Einsprüchen ziehe ich die Möglichkeit einer nicht-diskriminierenden, embryopathischen Begründung solcher Schwangerschaftsabbrüche in Zweifel. Dagegen stimme ich Tarkian in ihrer allgemeinen Einschätzung zu, dass Schwangerschaftsabbrüche, die in Reaktion auf eine pränatale Diagnose vorgenommen werden, nicht per se diskriminierend sind

\footnotetext{
${ }^{4}$ Im Hintergrund der Rede von „dem, worum es hier und jetzt für mich eigentlich ginge“, steht der Gedanke einer „Forderung der Stunde“, den ich von Max Scheler übernehme (vgl. Scheler 1927, 513) und in meinem bereits erwähnten Aufsatz über „Das Lieben in der Geburt“ (i. V.) näher ausführe.
} 
und dass in ihnen zugleich selbstbestimmtes Gebären ausgeübt werden kann. Auf Formen von Schwangerschaftsabbrüchen, die in Reaktion auf PND ausgeübt werden, ohne deswegen diskriminierend zu sein, werde ich am Ende meines Kommentars zurückkommen. Die These, dass in bestimmten dieser nicht-diskriminierenden Schwangerschaftsspätabbrüchen eine besondere Form des selbstbestimmten Gebärens ausgeübt werden kann, kann ich im Rahmen des vorliegenden Kommentars aus Platzgründen nicht erörtern.

Tarkian hat ihre Fokussierung auf die embryopathische Begründung nicht näher erläutert. Meines Erachtens speist sie sich aus einem Ineinandergreifen ihres Leitprinzips der reproduktiven Handlungsfreiheit und der besonderen Verfasstheit ihres Erkenntnisobjekts: der Schwangerschaftsabbrüche in Reaktion auf eine bestimmte Diagnose durch PND. Da sie nach solchen Schwangerschaftsabbrüchen in Reaktion auf PND fragt, die die reproduktive Handlungsfreiheit erweitern, scheiden für sie all die Fälle aus, in denen die Schwangere bei der Entscheidung zum Abbruch keine eigenen Projekte verfolgt, sondern äußerem Zwang - insbesondere vorherrschender Diskriminierung oder fehlender Inklusion - unterliegt. Da sich die Schwangerschaftsabbrüche in Reaktion auf PND überdies dadurch von anderen Schwangerschaftsabbrüchen unterscheiden, dass die Schwangere in ihrer aktuellen Lebenssituation ein Kind ,prinzipiell“ gewollt hätte (vgl. Rehmann-Sutter in Vorbereitung), bleibt für Tarkian nur ein Fall übrig, in dem diese Form des Abbruchs eine Erweiterung der reproduktiven Freiheit darstellen kann: nämlich der Fall, in dem die Schwangere ihre Schwangerschaft beendet, weil das Kind schwer erkrankt oder behindert ist (vgl. Tarkian, Abschnitt 6).

Für meine Frage nach der Willensfreiheit in solchen Schwangerschaftsabbrüchen ist es nun entscheidend, ob Tarkians Argument überzeugen kann, dass in der embryopathischen Begründung keine ,ganz grundsätzlich[e]“ „Diskriminierung von Menschen mit [den] Merkmalen“ vorliegen müsse, ,gegen die pränatal selegiert wird“ (ebd.). Dabei stellt sich Tarkian erneut gegen den Geist der aktuellen Gesetzeslage in Deutschland. Zur Anwendung kommt in diesem Zusammenhang eine gesetzliche Regelung der Schwangerschaftsspätabbrüche, da die entscheidenden pränataldiagnostischen Untersuchungen erst nach der 12. Schwangerschaftswoche vorgenommen werden können (vgl. Weilert in Vorbereitung). Vor dem Hintergrund der Kritik von Kirchen und Behindertenverbänden an der embryopathischen Begründung als diskriminierend wurde der Schwangerschaftsspätabbruch in den 1990er Jahren gesetzlich - in $§ 218$ a (2) StGB - neu geregelt. Seither werden Schwangerschaftsspätabbrüche in Deutschland nur mit medizinischer Indikation als ,nicht rechtswidrig“ eingestuft: ,wenn der Abbruch der Schwangerschaft unter Berücksichtigung der gegenwärtigen und zukünftigen Lebensverhältnisse der Schwangeren nach ärztlicher Erkenntnis angezeigt ist, um eine Gefahr für das Leben oder die Gefahr einer schwerwiegenden Beeinträchtigung des körperlichen oder seelischen Gesundheitszustandes der Schwangeren abzuwenden, und die Gefahr nicht auf eine andere für sie zumutbare Weise abgewendet werden kann“ ( $\$ 281$ a (2) StGB).

Die Einstufung der ,pränatalen Selektion gegen zukünftige Kinder“ als diskriminierend weist Tarkian mit dem Argument fehlender Differenziertheit zurück und verlangt eine kasuistische Unterscheidung (vgl. ebd.). Tarkian zufolge ,variiert“ die Plausibiliät des Diskriminierungsarguments ,,mit möglichen Merkmalen zukünftiger 
Kinder, gegen die selegiert werden könnte. Es gibt einige schwere erbliche Stoffwechselerkrankungen, die bislang nicht ursächlich therapierbar sind und zu einer deutlich reduzierten Lebenserwartung der Betroffenen führen“ (ebd.). „In diesen seltenen Fällen geht es" nach Tarkian nicht um eine Diskriminierung des Kindes, sondern vielmehr ,darum, ein schweres Übel von jemandem abzuwenden, welches auf keine andere Weise abgewendet werden kann als dadurch, dass seine Existenz verhindert wird“" (Tarkian, Abschnitt 7). Implizit nimmt sie in diesem Schluss die Prämisse in Anspruch, dass bestimmte schwere, bisher nicht therapierbare Erkrankungen und Behinderungen das Leben des Kindes zu einem „schweren Übel“ machten, sodass es nicht mehr lebenswert sei. Da die embryopathische Begründung eines Schwangerschaftsabbruchs nicht notwendigerweise auf einer diskriminierenden Haltung gegenüber kranken und behinderten Menschen, sondern auch auf dem rationalen Urteil basieren könne, dass das kindliche Leben mit bestimmten Erkrankungen oder Behinderungen nicht lebenswert sei, sei, so Tarkian, eine Einschränkung der reproduktiven Freiheit in diesen Fällen moralisch nicht geboten. Vielmehr sei der Ausbau von Beratungsangeboten zu fordern, ,um Vorurteilen oder Fehleinschätzungen über den Lebensalltag von Menschen mit Behinderung entgegenzuwirken“ (ebd.) - und die Betroffenen in ihrer Ausübung ihrer reproduktiven Handlungsfreiheit $\mathrm{zu}$ unterstützen.

Auf die erkenntnistheoretischen Schwierigkeiten des Gedankens, jemanden vor einem Übel zu bewahren, indem man seine Existenz verhindere, möchte ich an dieser Stelle nicht näher eingehen. Meines Erachtens wird dieser Gedanke von derselben Schwierigkeit wie Thomas Nagels Gedanke eingeholt, dass der Tod uns mit dem Leben eines Gutes beraube (vgl. Nagel 2008, 18): dass er - in den Worten von Ernst Tugendhat - ,implizier[t], daß das Subjekt [...] irgendwie erhalten bleibt, während es doch in Wirklichkeit aufhört zu existieren“ (Tugendhat 2006, 26). Vielmehr möchte ich meine Einsprüche an Tarkians Annahme richten, dass ein rationales Urteil über den Wert oder Unwert des kindlichen Lebens mit einer schweren Erkrankung oder Behinderung möglich sei. Tarkians Annahme zieht ihre Plausibilität aus den Extremsituationen, die beurteilt werden: dass es Extremsituationen schrecklicher Erkrankungen oder Behinderungen gebe, die für einen rationalen Standpunkt nur das Urteil zuließen, dass es für das Kind besser sei, erst gar nicht geboren zu werden. Die besondere Verfasstheit seines Gegenstandes ändert jedoch nichts am erkenntnistheoretischen Status solch eines Urteils: es ist ein Werturteil, das wir inmitten des Lebens fällen. Inmitten des Lebens bzw. unter den Bedingungen der Zeit verfügen wir jedoch über keinen Erkenntnisstandpunkt, um den Wert eines Lebens, mit oder ohne welche Erkrankung und Behinderung auch immer, rational zu beurteilen. Wir verfügen über kein allgemeingültiges Wissen darüber, was ein Leben gut, glücklich oder sinnvoll macht. Wir wissen nicht, ob in wenigen Jahren nicht doch eine Therapiemöglichkeit gefunden wird, die heute niemand erahnt hätte; wir können auch die Glücks- und Sinnpotenziale nicht bemessen, die ein Leben in Situationen höchster Bedrängnis und größten Leidens eröffnen kann. Umgekehrt wissen wir auch nicht, welches Leiden im Leben eines Kindes bevorsteht, das während der Schwangerschaft - noch ?? - nicht krank oder behindert ist. Wenn wir unter den Bedingungen der Zeit gleichwohl Werturteile über den Wert oder Unwert des Lebens fällen, das einem Kind bevorsteht, dann sind diese Urteile notwendigerweise 
politischer Natur. In ihnen nehmen wir Idealvorstellungen eines guten Lebens und die damit einhergehenden normalisierenden Ressentiments in Anspruch, die wir in unserer Vergangenheit - unter äußerem Einfluss oder nicht - ausgebildet haben. Damit kollabiert im Fall eines embryopathisch begründeten Schwangerschaftsabbruchs aber der Unterschied zwischen Diskriminierung und sachlicher Beurteilung des Werts des zukünftigen Lebens eines Kindes. Indem darin „Leitbilder“ (Kersting 2017) eines wertvollen oder unwerten Lebens in Anspruch genommen werden, ist ein embryopathisch begründeter Schwangerschaftsabbruch immer diskriminierend. Dergestalt verletzt er die Würde des Kindes und macht zugleich einen Akt des Gebärens aus, in dem wir in unserem Wollen nicht frei sind, sondern von unseren eigenen emotionalen Verstrickungen getrieben werden - und zwar selbst dann, wenn wir in unserem Handeln frei zur Ausübung einer derartigen Ausgestaltung unserer Fortpflanzung wären, was wir unter der bestehenden Gesetzeslage in Deutschland gegenwärtig nicht sind.

Auch wenn ich eine embryopathische Begründung - aufgrund ihres problematischen Status in der Zeit - für weit problematischer halte als Tarkian, teile ich gleichwohl ihre Einschätzung, dass nicht jede Entscheidung zu einem Schwangerschaftsabbruch in Reaktion auf den pränataldiagnostischen Befund einer schweren Erkrankung oder Behinderung des Kindes diskriminierend sein muss. Entscheidungen, sich PND-Tests zu unterziehen und die Schwangerschaft in Reaktion auf eine schwere Erkrankung oder Behinderung des Kindes zu beenden, müssen nämlich nicht embryopathisch begründet sein - ohne deswegen freilich immer auch Akte der Freiheit zu formen. Dies zeigt ein Blick auf Schwangerschaftsabbrüche mit medizinischer Indikation, die gegenwärtig in Deutschland als „,nicht rechtswidrig“ eingestuft werden. In Fällen, in denen in der PND Erkrankungen an Mutter und Kind festgestellt werden, die das Leben der Schwangeren gefährden bzw. ihre körperliche Gesundheit stark beeinträchtigen würden und bei denen diese Gefahr nur durch den Abbruch der Schwangerschaft abgewendet werden kann, ist dieser Eingriff ganz offensichtlich nicht diskriminierend. Dem Abbruch liegt dann nämlich gar kein Urteil über den Wert des kindlichen Lebens, sondern über die Überlebensmöglichkeiten der Mutter zugrunde. Damit ist ein solcher Abbruch weder entwürdigend für das Kind noch Ergebnis eines Ressentiment-getriebenen Wollens der Mutter. Dagegen mag es als fraglich erscheinen, ob das Problem des mütterlichen VerhaftetSeins in Ressentiments gegenüber Menschen mit Behinderung in den Fällen der psychologischen Indikation nur verschleiert, nicht jedoch unterlaufen wird: wenn das Leiden seelischer Natur ist, das der Mutter von einer diagnostizierten Erkrankung oder Behinderung des Kindes erwächst und durch einen Abbruch der Schwangerschaft behoben werden soll (vgl. Rothhaar in Vorbereitung). Hier ist nochmals zwischen gegenwärtigem und künftigem seelischem Leiden der Mutter zu unterscheiden. In Bezug auf eine aktuelle, ,,schwerwiegende[...] Beeinträchtigung des [...] seelischen Gesundheitszustandes der Schwangeren", die allein durch den Abbruch der Schwangerschaft abzuwenden ist, ließen sich zahlreiche Fälle erdenken, die keinen Ressentiments gegenüber Menschen mit Behinderung verhaftet sind. So könnte etwa die Schwangere selbst Trägerin der schweren Erkrankung sein, die an ihrem Kind diagnostiziert wurde, und nun von der Erfahrung eingeholt werden, mit ihren Bemühungen der Familiengründung schwere Schuld auf sich geladen zu haben: von 
Erfahrungen, an denen sich schwere Depressionen entzünden können und die u.U. nur durch den Abbruch der Schwangerschaft behoben werden können. ${ }^{5}$ Aber wie sieht es in Bezug auf seelisches Leid aus, das die Mutter erst für ihr künftiges Leben mit dem erkrankten oder behinderten Kind erwartet - und das in ihr schon jetzt den Wunsch entstehen lässt, ihre Schwangerschaft abzubrechen, um diese Beeinträchtigung ihrer psychischen Gesundheit abzuwenden? Zehrt die Prognose künftigen Leidens nicht notwendigerweise von dem Ressentiment geleiteten Werturteil, dass das versehrte Leben des Kindes ein Übel für sich und seine Mutter darstellen werde? Meines Erachtens kann dies, muss jedoch nicht der Fall sein. Es kann sein, dass die Mutter in der Gegenwart künftiges, seelisches Leiden befürchtet, weil sie - geleitet von Ressentiments - die Erkrankung oder Behinderung ihres Kindes als ein Übel beurteilt, das ihr künftiges, mit dem Kind geteiltes Leben für sie zu einer unerträglichen Last formen wird. Damit bestünde die Gefahr, dass unter dem Deckmantel der medizinischen Indikation ein embryopathisch begründeter Schwangerschaftsabbruch vorgenommen würde (vgl. Rothhaar in Vorbereitung). Es kann aber auch sein, dass die Schwangere künftiges, seelisches Leid ganz anderer Natur befürchtet: künftiges Leid, das ihr aus einer Entscheidung in der Gegenwart entwächst, mit der sie an sich selbst ,,vorbeigelebt“ hat, ${ }^{6}$ d. h. mit der Entscheidung, an der Schwangerschaft festzuhalten, obwohl es in der Gegenwart für sie darum gegangen wäre, die Schwangerschaft zu beenden - etwa, um keine Schuld auf sich zu ziehen. In solchen Fällen fällt eine schwangere Frau wiederum kein Werturteil über den Wert des kindlichen Lebens, sondern über ihre eigenen Möglichkeiten, unter ihren konkreten Lebensbedingungen jetzt und in Zukunft so zu leben, wie es von ihr verlangt ist - um gut für sich selbst, das Kind und ihre weiteren Angehörigen zu sorgen (vgl. Rehmann-Sutter in Vorbereitung). Für diese Einschätzung ihrer eigenen Möglichkeiten, ihr individuelles Selbst-Sein und ihr Mutter-Sein eines schwer erkrankten oder behinderten Kindes gut auszuüben, sind die Formen der Unterstützung zentral, die sie zu erwarten hat. Aus diesem Grund möchte ich am Ende meiner Überlegungen Tarkian in ihrer Forderung nach dem Ausbau von gesamtgesellschaftlicher Inklusion nachdrücklich zustimmen.

Funding Open Access funding provided by Projekt DEAL.

Open Access Dieser Artikel wird unter der Creative Commons Namensnennung 4.0 International Lizenz veröffentlicht, welche die Nutzung, Vervielfältigung, Bearbeitung, Verbreitung und Wiedergabe in jeglichem Medium und Format erlaubt, sofern Sie den/die ursprünglichen Autor(en) und die Quelle ordnungsgemäß nennen, einen Link zur Creative Commons Lizenz beifügen und angeben, ob Änderungen vorgenommen wurden.

Die in diesem Artikel enthaltenen Bilder und sonstiges Drittmaterial unterliegen ebenfalls der genannten Creative Commons Lizenz, sofern sich aus der Abbildungslegende nichts anderes ergibt. Sofern das betreffende Material nicht unter der genannten Creative Commons Lizenz steht und die betreffende Handlung nicht nach gesetzlichen Vorschriften erlaubt ist, ist für die oben aufgeführten Weiterverwendungen des Materials die Einwilligung des jeweiligen Rechteinhabers einzuholen.

\footnotetext{
${ }^{5}$ Zum Verhältnis von Erfahrungen des Schuldig-Werdens und der Ausbildung von Depressionen vgl. Fuchs 2008, $163 \mathrm{f}$.

${ }^{6}$ Zur Erfahrung des „An-sich-Vorbei-Lebens“ und des daraus erwachsenden Leidens vgl. Fuchs 2019, $90 \mathrm{ff}$.
} 
Weitere Details zur Lizenz entnehmen Sie bitte der Lizenzinformation auf http://creativecommons.org/ licenses/by/4.0/deed.de.

\section{Literatur}

Fuchs, Thomas. 2008. Existenzielle Vulnerabilität. Ansätze zu einer Psychopathologie der Grenzsituationen. In: Leib und Lebenswelt. Neue philosophisch-psychiatrische Essays, 148-171. Zug. Die graue Edition.

Fuchs, Thomas. 2019. Versöhnung mit dem Ungelebten. Zum Gelingen des Lebens im Sterben. In Gelingendes Sterben. Zeitgenössische Theorien im interdisziplinären Dialog, hrsg. von Olivia Mitscherlich-Schönherr, 85-100. Berlin. de Gruyter.

Kersting, Daniel. 2017. Tod ohne Leitbild? Philosophische Untersuchungen zu einem integrativen Todeskonzept. Münster. mentis.

Mitscherlich-Schönherr, Olivia. (i. V.). Das Lieben in der Geburt. Eine verstehende Liebesethik des ZurWelt-Bringens von Kindern. In Das Gelingen der Geburt. Zeitgenössische Theorien im interdisziplinären Dialog, hrsg. von Reiner Anselm und Olivia Mitscherlich-Schönherr. Berlin. de Gruyter.

Nagel, Thomas. 2008. Der Tod. In: Letzte Fragen. Moral Questions, 17-28. Hamburg. Europäische Verlagsanstalt.

Plessner, Helmut. 1975. Die Stufen des Organischen und der Mensch. Einleitung in die philosophische Anthropologie. Berlin/New York. de Gruyter.

Rehmann-Sutter, Christoph. (i. V.). Zur ethischen Bedeutung der vor-geburtlichen Diagnostik. In Das Gelingen der Geburt, hrsg. von Reiner Anselm und Olivia Mitscherlich-Schönherr. Berlin. de Gruyter.

Rothhaar, Markus. (i. V.). Gerechtfertigter Fetozid? Eine rechtsphilosophische Kritik von Spätabbrüchen. In Das Gelingen der Geburt, hrsg. von Reiner Anselm und Olivia Mitscherlich-Schönherr. Berlin. de Gruyter.

Scheler, Max. 1927. Der Formalismus in der Ethik und die materiale Wertethik, Halle. Max Niemeyer.

Schües, Christina. 2003. Moralphilosophische Fragen zum Embryo. Am Anfang ist die Beziehung. In Der Traum vom besseren Menschen. Zum Verhältnis von praktischer Philosophie und Biotechnologie, hrsg. von Rudolf Rehn, Christina Schües und Frank Weinreich, 33-54.Frankfurt a. Main u. a. Peter Lang.

Schües, Christina. (i. V.). Das Versprechen der Geburt. In Das Gelingen der Geburt, hrsg. von Reiner Anselm und Olivia Mitscherlich-Schönherr. Berlin. de Gruyter.

Staehler, Tanja. 2016. Vom Berührtwerden. Schwangerschaft als paradoxes Phänomen. In Dem Erleben auf der Spur. Feminismus und die Philosophie des Leibes, hrsg. von Hilge Landwehr und Isabella Marcinski, 27-44. Berlin. transcript.

Staehler, Tanja. (i. V.). Umkehrungen. Wie Schwangerschaft und Geburt unsere Welterfahrungen auf den Kopf stellen. In Das Gelingen der Geburt, hrsg. von Reiner Anselm und Olivia Mitscherlich-Schönherr. Berlin. de Gruyter.

Tugendhat, Ernst. 2006. Über den Tod. Frankfurt a. Main. Suhrkamp.

Weilert, Katarina. (i.V.). Die Grundrechtsstellung des extrauterin lebensfähigen Fötus in Spannung zu den Grundrechten seiner Eltern. In Das Gelingen der Geburt, hrsg. von Reiner Anselm und Olivia Mitscherlich-Schönherr. Berlin. de Gruyter.

Wiesemann, Claudia. 2006. Von der Verantwortung, ein Kind zu bekommen. Eine Ethik der Elternschaft. München. C.H. Beck. 